\title{
A Prospective Comparison of Selective Multiparametric Magnetic Resonance Imaging Fusion-Targeted and Systematic Transrectal Ultrasound-Guided Biopsies for Detecting Prostate Cancer in Men Undergoing Repeated Biopsies
}

\author{
Lars Boesen ${ }^{\mathrm{a}}$ Nis Nørgaard ${ }^{\mathrm{a}} \quad$ Vibeke Løgager $^{\mathrm{b}} \quad$ Ingegerd Balslev $^{\mathrm{c}}$ \\ Henrik S. Thomsen ${ }^{b}$ \\ ${ }^{a}$ Department of Urology, ${ }^{b}$ Department of Radiology and ${ }^{\mathrm{C}}$ Department of Pathology, Herlev Gentofte University \\ Hospital, Herlev, Denmark
}

\section{Keywords}

Prostate cancer · Biopsy · Magnetic resonance imaging ·

Ultrasonography · Outcome

\begin{abstract}
Introduction: The aim of the study was to compare the prostate cancer ( $\mathrm{PCa}$ ) detection rate of systematic transrectal ultrasound-guided biopsies (TRUS-bx) and multiparametricMRI targeted biopsies (mp-MRI-bx) in a repeat biopsy setting and evaluate the clinical significance following an "MRI-targeted-only" approach. Materials and Methods: Patients with prior negative biopsies underwent prostatic multiparametric-MRI that was scored using the Prostate Imaging Reporting and Data System (PI-RADS) classification. All underwent both repeated TRUS-bx and mp-MRI-bx using image fusion of any PI-RADS $\geq 3$ lesion. Biopsy results from TRUS-bx, mp-MRI-bx, and the combination were compared. Results: PCa was detected in 89 out of $206(43 \%)$ patients. Of these, $64(31 \%)$ and 74 (36\%) patients were detected using mpMRI-bx and TRUS-bx, respectively. Overall, mp-MRI-bx detected fewer patients with low-grade (Gleason score [GS] $3+$ 3) cancers (14/64 vs. $41 / 74)$ and more patients with interme-
\end{abstract}

(c) 2017 S. Karger AG, Basel

\section{E-Mail karger@karger.com} www.karger.com/uin diate/high-grade cancers (GS $\geq 3+4)(50 / 64$ vs. $33 / 74)$ using fewer biopsy cores compared with TRUS-bx $(p<0.001)$. Using an "MRI-targeted-only" approach in men with PI-RADS $\geq 3$ lesions reduced the number of men requiring repeated biopsies by $50 \%$, decreased low-grade cancer diagnoses by $66 \%$, and increased intermediate/high-grade cancer diagnoses by $52 \%$. Conclusions: MRI-targeted biopsies have a high detection rate for significant PCa in patients with prior negative transrectal ultrasound-guided biopsies and preferentially detect intermediate/high-grade compared with low-grade tumors.

(c) 2017 S. Karger AG, Basel

\section{Introduction}

Transrectal ultrasound-guided biopsies (TRUS-bx) have limited diagnostic accuracy in detecting prostate cancer $(\mathrm{PCa})$ and often fail to diagnose significant cancers [1]. To overcome this problem, patients with negative TRUSbx regularly undergo repeated biopsy procedures with some urologists recommending increasing the number of cores [2], while others suggest saturation biopsy tech- 
niques [3]. These approaches often lead to an increased PCa detection rate, but also increase the diagnosis of insignificant low-grade tumors potentially leading to unnecessary treatment plans [4-6]. PCa is evaluated using the Gleason score (GS), which is strongly related to tumor aggressiveness and prognosis. Pre-therapeutic risk assessments based on the GS from TRUS-bx can be inaccurate due to sampling errors. This is confirmed by an upgraded GS in one third of patients following radical prostatectomy (RP) [7]. Inaccurate GS at biopsy may lead to incorrect risk stratification and possibly to over- or under-treatment.

The limitations of TRUS-bx have highlighted the need for improved diagnostic tools, such as biomarkers, or imaging techniques that might enhance the identification of significant PCa without increasing the detection of insignificant tumors. Better techniques could possibly decrease the number of unnecessary biopsy sessions and cores.

Multiparametric MRI (mp-MRI) and targeted biopsies can improve the detection of significant PCa [8-12] and lead to more accurate GS grading [13-15]. Fusion software combining mp-MRI data and real-time TRUS imaging has been developed to increase the accuracy of targeted biopsies and can now be used in outpatient clinics. There is an ongoing debate question whether targeted biopsies should be used in isolation or in combination with systematic biopsies to increase the diagnostic yield of significant high-grade cancers while excluding lowergrade tumors. In this prospective study, we address this dilemma by comparing the detection rate of PCa by $\mathrm{mp}$ MRI/TRUS fusion-targeted biopsies (mp-MRI-bx) with the detection rate of systematic TRUS-bx in the same patient cohort, which had prior negative TRUS-bx results. In addition, we evaluated the significance of the detected cancers and compared the results of an "mp-MRI-targeted-only" and a systematic TRUS-bx approach.

\section{Materials and Methods}

This prospective trial was approved by the Local Committee for Health Research Ethics (No. H-1-2011-066) and the data protection agency. It was registered at Clinicaltrials.gov (No. NCT01640262). All patients were prospectively enrolled between September 2012 and September 2013 after they gave written informed consent. Inclusion criteria required that all had at least one prior negative TRUS-bx session (10-12 cores) and a persistent clinical suspicion of PCa (elevated PSA, an abnormal digital rectal examination, or a previous abnormal TRUS image) that warranted a repeat biopsy (rebiopsy). The exclusion criteria were a prior $\mathrm{PCa}$ diagnosis, prior prostate $\mathrm{mp}-\mathrm{MRI}$, or presence of general contraindications for MRI.

MRI Fusion-Targeted Prostate Biopsies

\section{Multiparametric MRI}

Mp-MRI was performed prior to rebiopsy using a 3.0 T MRI magnet (Ingenia, Philips Healthcare, Best, The Netherlands) with a pelvic-phased-array coil (Philips Healthcare) positioned over the pelvis. Tri-planar T2-weighted-, diffusion-weighted-, and dynamic contrast-enhanced images according to the European Society of Urogenital Radiology [9] recommendation and as previously described [15].

All mp-MRI images were reviewed by the same dedicated $\mathrm{mp}$ MRI physician with 2 years of experience in prostate interpretation and all suspicious lesions were registered on an 18-region modified prostate diagram provided by the European Consensus Meeting [16]. All patients were overall classified by the Prostate Imaging Reporting and Data System (PI-RADS) version 1 classification [9] on a 5-point PI-RADS scale (1-highly unlikely, 2-unlikely, 3-equivocal, 4-likely, and 5-highly likely) based on their likelihood of having clinically significant PCa. Patients with no suspicious lesions were classified as PI-RADS score 1 . The newly published PI-RADS version 2 [17] was not available during the timeframe of this study.

\section{Biopsy: TRUS-bx and $m p-M R I-b x$}

Initially, all patients underwent systematic repeated TRUS-bx blinded to mp-MRI findings. This included a 10-core extended sextant re-biopsy-scheme from the lateral and medial part of the prostate (base, mid, apex) on both the left and right sides. Abnormalities on TRUS-bx were sampled using the standard core for the relevant segment. TRUS-bx was immediately followed by targeted mp-MRIbx of any identified lesion using the HI-RVS-system (Hitachi Ltd., Tokyo, Japan). This system uses a small electromagnetic field generator placed in close proximity to the patient and tracks the spatial location of the TRUS probe using a small attached sensor. Patient mp-MRI data were loaded into the system after TRUS-bx and fused and synchronized with the corresponding TRUS images using zonal anatomy and tissue landmarks. Mp-MRI-bx was targeted toward mp-MRI-identified lesions (1-2 cores/lesion) using T2-weightedimaging superimposed on the real-time TRUS images. All prostate biopsies (TRUS-bx and mp-MRI-bx) were taken in the axial plane using an end-fire TRUS probe and were performed by the same operator who had extensive experience in TRUS-bx ( $>20$ years) but less experience in software-based image fusion (1 year).

\section{Histopathological Evaluation}

All biopsy samples were described by the same genitourinary pathologist with $>11$ years of dedicated experience. The location on the prostate diagram, the GS based on the International Society of Urological Pathology 2005 consensus [18], and the quantity of cancerous tissue per core (\%) were all determined for each PCapositive biopsy core. The patients GS scores were divided into 3 categories: low $(\mathrm{GS} \leq 6)$, intermediate $(\mathrm{GS}=7)$, and high $(\mathrm{GS} \geq 8$ ) grade $\mathrm{PCa}$. In addition, patients were allocated to International Society of Urological Pathology 2014 Gleason grade groups [19] based on the GS scoring criteria [18]. Cancer significance was defined as (1) insignificant low-grade PCa (GS 6) and (2) significant intermediate/high-grade PCa (GS $\geq 7$ ).

\section{Statistical Analysis}

Patient characteristics were stratified by biopsy results and assessed using descriptive statistics. Continuous variables including age, PSA, PSA-d, prior biopsy procedures, number of mp-MRI lesions, and number of mp-MRI-bx were compared using the Wil- 
Table 1. Patient characteristics

\begin{tabular}{|c|c|c|c|c|}
\hline & $\begin{array}{l}\text { Total } \\
(n=206)\end{array}$ & $\begin{array}{l}\text { PCa negative } \\
(n=117)\end{array}$ & $\begin{array}{l}\text { PCa positive } \\
(n=89)\end{array}$ & $p$ value \\
\hline Age, years, median (IQR) & $65(58-68)$ & $63(47-67)$ & $66(61-99)$ & 0.304 \\
\hline PSA, ng/mL, median (IQR) & $12.8(8.9-19.6)$ & $11.0(7.9-17.1)$ & $14.0(9.7-21.7)$ & 0.032 \\
\hline PSA density, ng/mL/mL, median (IQR) & $0.20(0.13-0.29)$ & $0.16(0.12-0.23)$ & $0.26(0.17-0.45)$ & 0.001 \\
\hline Prior biopsy, median (IQR) & $2(2-3)$ & $2(2-3)$ & $2(1-3)$ & 0.124 \\
\hline Time $_{\mathrm{mp}-\mathrm{MRI}}$ to biopsy, days & $7(1-14)$ & $7(1-15)$ & $2(0-14)$ & 0.527 \\
\hline \multicolumn{5}{|l|}{$\mathrm{cT}_{\text {DRE }}$ category, $n(\%)$} \\
\hline Nonpalpable lesions (cT1) & $188(91)$ & $110(94)$ & $78(88)$ & 0.137 \\
\hline Palpable lesions (cT2-T3) & $18(9)$ & $7(6)$ & $11(12)$ & \\
\hline \multicolumn{5}{|l|}{$\mathrm{cT}_{\text {TRUS }}$ category, $n(\%)$} \\
\hline Nonvisual lesions (cT1) & 141 & 89 & 52 & \\
\hline Visual lesions (cT2-T3) & 65 & 28 & 37 & 0.010 \\
\hline Mp-MRI included lesions, $n$ & 302 & 209 & 93 & \\
\hline Lesion/patient, mean (range) & $1.5(1-4)$ & & & \\
\hline \multicolumn{5}{|l|}{ Zone of origin, $n(\%)$} \\
\hline Peripheral zone & $155(51)$ & 116 & 38 & \\
\hline Transitional zone/anterior location & $147(49)$ & 93 & 55 & 0.025 \\
\hline
\end{tabular}

PCa, prostate cancer; TRUS, transrectal ultrasound; mp-MRI, multiparametric MRI; IQR, interquartile range.

coxon Rank sum test. Fisher's exact test was used to compare the tumor stage determined by digital rectal examination $\left(\mathrm{cT}_{\mathrm{DRE}}\right)$ and TRUS ( $\mathrm{cT}_{\text {TRUS }}$ ) pooled in non-palpable/palpable and nonvisual/visual tumor groups including the zone of lesion origin. Pre-biopsy mp-MRI PI-RADS scores were compared with biopsy results using a chi-square analysis to determine the correlation between suspicion on mp-MRI and positive biopsies. PCa-detection rates using mp-MRI-bx and systematic TRUS-bx were compared using the McNemar test. The highest GS from systematic TRUS-bx and mpMRI-bx from each patient were compared and examined using a paired $t$ test and further evaluated for accuracy using weighted kappa-statistics. A $p$ value below 0.05 was considered significant. Statistical analyses were performed using SPSS software version 20.0 (SPSS Inc., Chicago, IL, USA).

\section{Results}

Demographic data are displayed in Table 1. Of 213 prospectively enrolled patients, 7 were excluded because of mp-MRI technical problems or because they were claustrophobic. PCa was detected in 89 out of $206(43 \%)$ patients and the remaining $(57 \%)$ had a benign condition $(n=107)$, HGPIN $(n=2)$, or cellular changes indicating adenocarcinoma $(n=8)$.

\section{TRUS- $b x$}

Abnormal TRUS image results were observed in 65 (32\%) patients and TRUS-bx-detected PCa in $74(36 \%)$ of the 206 patients. Of these, 41 (55), 28 (38), and 5 (7\%) had had low- $(\mathrm{GS} \leq 6)$, intermediate- (GS =7), and high-grade $(G S \geq 8) P C a$, respectively. Most patients $(86 \%)$ had predominantly GS grade $3(3+3$ or $3+4)$ corresponding to Gleason grade groups 1 or 2 .

\section{$M p-M R I-b x$}

Mp-MRI-bx was performed in 189 out of 206 (92\%) patients with PI-RADS scores $2-5$ and detected PCa in 64 out of 206 (31\%). Of these, 14 (22), 36 (56), and 14 (22\%) had low-, intermediate-, and high-grade PCa, respectively. The proportion of intermediate/high-grade PCa was significantly higher using mp-MRI-bx (Table $2, p<0.001$ ) and there was a lower PCa detection rate in patients with PI-RADS 1-2 compared with PI-RADS 4-5 (20 vs. 75\%, $p<0.001$; Fig. 1). Mp-MRI were without lesions in 17 out of 206 patients who did not have mp-MRI-bx. TRUS-bxdetected PCa in 4 of these patients and all had 1 out of 10 positive cores with GS $6(3+3)$ in $5-10 \%$ of the biopsy length.

\section{$M p-M R I-b x v s . T R U S-b x$}

Of the 89 patients with $\mathrm{PCa}, 25$ patients were detected only using TRUS-bx, 15 patients were detected only using mp-MRI-bx, and 49 patients were detected using both methods. Although TRUS-bx detected more patients with $\mathrm{PCa}$, the difference was not statistically significant (McNemar, $p=0.155)$. Mp-MRI-bx cores had a greater mean cancerous core length $(43.3$ vs. $22.4 \%, p<0.001)$ 
Table 2. Comparison of the GS and grade for patients between mp-MRI-bx and systematic TRUS-bx

\begin{tabular}{|c|c|c|c|c|c|c|c|}
\hline & \multicolumn{7}{|c|}{ TRUS-bx } \\
\hline & no PCa & $\begin{array}{l}\text { GS } 6 \\
\text { grade } 1\end{array}$ & $\begin{array}{l}\text { GS } 7(3+4) \\
\text { grade } 2\end{array}$ & $\begin{array}{l}\text { GS } 7(4+3) \\
\text { grade } 3\end{array}$ & $\begin{array}{l}\text { GS } 8 \\
\text { grade } 4\end{array}$ & $\begin{array}{l}\text { GS } 9-10 \\
\text { grade } 5\end{array}$ & total \\
\hline \multicolumn{8}{|l|}{ Mp-MRI-bx } \\
\hline No PCa & 0 & 23 & 2 & 0 & 0 & 0 & 25 \\
\hline GS 6 grade 1 & 4 & 9 & 1 & 0 & 0 & 0 & 14 \\
\hline GS $7(3+4)$ grade 2 & 5 & 6 & 14 & 1 & 0 & 0 & 26 \\
\hline GS $7(4+3)$ grade 3 & 2 & 2 & 4 & 2 & 0 & 0 & 10 \\
\hline GS 8 grade 4 & 3 & 1 & 2 & 2 & 2 & 0 & 10 \\
\hline GS $9-10$ grade 5 & 1 & 0 & 0 & 0 & 1 & 2 & 4 \\
\hline Total & 15 & 41 & 23 & 5 & 3 & 2 & 89 \\
\hline
\end{tabular}

GS, Gleason score; TRUS-bx, transrectal ultrasound-guided biopsies; mp-MRI-bx, multiparametric MRIguided biopsies.

Fig. 1. Patients diagnosed with prostate cancer (PCa) stratified by multiparametric MRI suspicion score (PI-RADS).

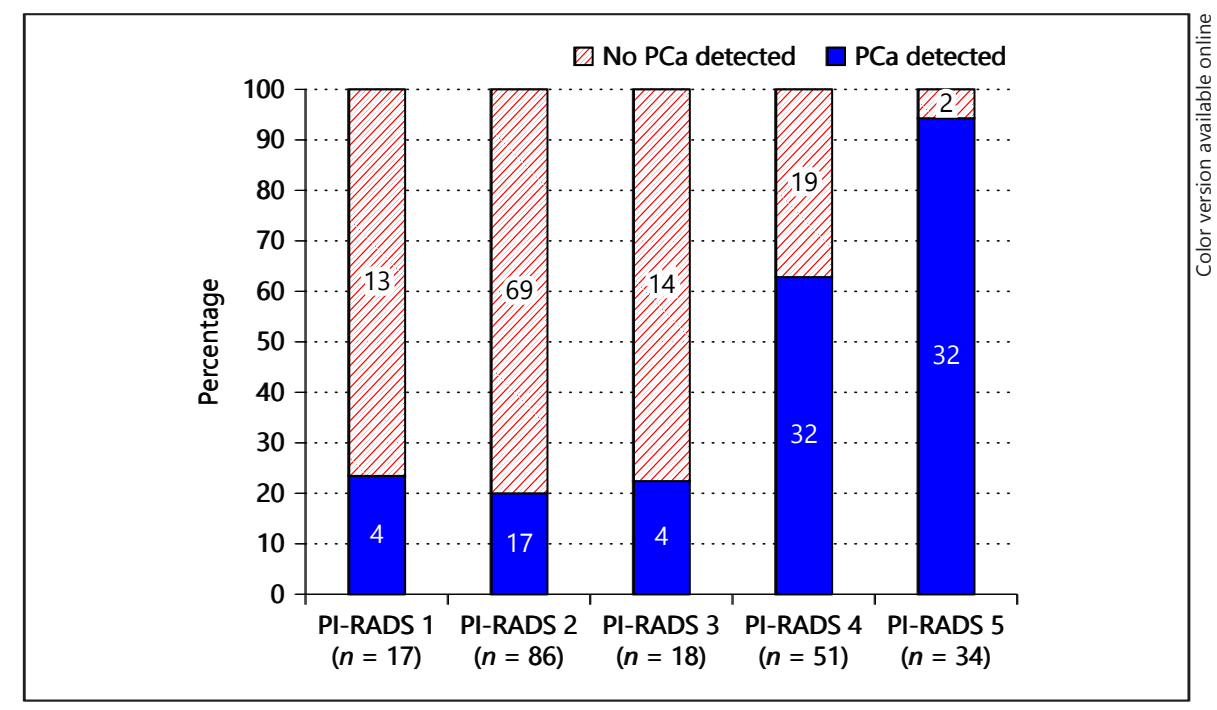

and a greater PCa detection yield per core $(28 \%$ of 421 targeted cores vs. $7.4 \%$ of 2,060 systematic cores, $p<$ 0.001) compared to TRUS-bx cores.

Overall, mp-MRI-bx detected 14 out of 89 (16\%) and TRUS-bx 41 out of 89 (46\%) patients with low-grade PCa $(p<0.001$; Fig. 2). Conversely, mp-MRI detected intermediate/high-grade PCa in 20 out of 89 (22\%) patients who were either missed $(n=11)$ or misclassified as low grade $(n=9)$ on TRUS-bx (Table 2). Equally, TRUS-bx detected significant cancer in 3 patients who were missed $(n=2)$ or misclassified as low grade $(n=1)$ on mp-MRIbx. Of the 15 patients (17\%) diagnosed with PCa only by mp-MRI-bx, 11 out of 15 (73\%) had significant intermediate/high-grade cancer. In PCa patients diagnosed both on TRUS-bx and mp-MRI-bx, 18 out of 49 (37\%) had an overall GS upgrade and 9 of these patients (50\%) were re-

classified from low-grade (GS 6) to significant intermediate/high-grade cancer based on the additional mp-MRIbx. The number of men requiring a repeat biopsy was reduced by $50 \%$, low-grade cancer diagnoses were decreased by $66 \%$, and intermediate/high-grade cancer diagnoses were increased by $52 \%$ if only patients with equivocal/highly suspicious lesions (PI-RADS $\geq 3$ ) followed an mp-MRI-'targeted only' approach without additional repeated TRUS-bx (Table 3).

\section{Discussion}

In this prospective study, we compared outcomes of mp-MRI fusion-targeted and systematic transrectal ultrasound-guided biopsies to detect PCa in men undergo- 


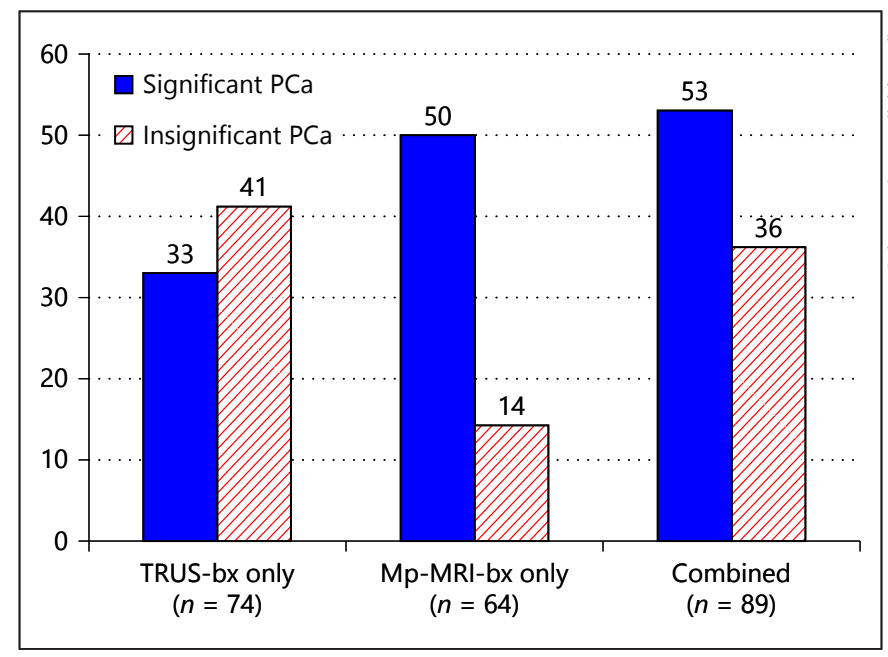

Fig. 2. Patients diagnosed with significant (Gleason score $\geq 3+4$ ) and insignificant (Gleason score $3+3$ ) prostate cancer $(\mathrm{PCa})$ stratified by biopsy technique (transrectal ultrasound-guided biopsies [TRUS-bx], multiparametric MRI-guided biopsies [mp-MRI-bx], and the combined approach).

Table 3. Per patient analysis of biopsy results comparing TRUS-bx and mp-MRI-bx for patients with PI-RADS score $\geq 3$

\begin{tabular}{lrrl} 
& TRUS-bx & Mp-MRI-bx & Difference, \% \\
\hline Biopsy patients, $n$ & 206 & 103 & -50 \\
Low-grade PCa & 41 & 14 & -66 \\
$\begin{array}{l}\text { Intermediate/high-grade } \\
\quad \text { PCa }\end{array}$ & 33 & 50 & 52 \\
Biopsy cores, $n$ & 2,060 & 209 & -90 \\
\hline
\end{tabular}

PCa, prostate cancer; TRUS-bx, transrectal ultrasound-guided biopsies; mp-MRI-bx, multiparametric MRI-guided biopsies; Low-grade PCa, Gleason score 6; intermediate/high-grade PCa, Gleason score $\geq 7$.

ing repeated biopsies and found an overall PCa detection rate of $43 \%(89 / 206)$ with $60 \%$ (53/89) harboring significant $(\mathrm{GS} \geq 7)$ cancers. We found that TRUS-bx detected more patients with PCa than mp-MRI-bx (36 vs. $31 \%$ ), although the difference was not statistically significant $(p=$ 0.155 ). However, the proportion of intermediate/highgrade PCa detected was significantly higher using mpMRI-bx, fewer biopsy cores were required, and these had a greater mean cancerous core-length. In addition, more than half of the patients with PCa detected using TRUSbx had low-grade disease. The poor PCa target identification of TRUS means clinicians are repeatedly faced with a dilemma in patients with negative TRUS-bx because a nonspecific rise in PSA is regarded as an indication for repeated prostate biopsies. Men considered at risk remain under PSA-surveillance for lengthy periods that involve repeated check-ups and possibly multiple biopsy sessions that can cause severe infections, bleeding, and anxiety. There is the additional problem of detecting insignificant low-grade PCa leading to possible overtreatment. Our findings are consistent with previous studies confirming that $\mathrm{mp}$-MRI and mp-MRI/TRUS-fusion biopsies can be used in this challenging patient group to identify undetected high-grade cancers in patients with prior negative TRUS-bx sessions $[12,14,20]$. Sonn et al. [12] demonstrated that office-based fusion biopsies can be performed in an outpatient clinic, thereby improving the detection of significant PCa in 105 patients, and Vourganti et al. [20] reported that only PSA density and mp-MRI-based suspicion were good indicators of significant cancer at repeat biopsy, whereas the number of previous biopsy sessions was not. We found a statistically significant correlation between the suspicion grade on $\mathrm{mp}-\mathrm{MRI}$ and detection of PCa on confirmatory biopsy $(p<0.001)$. Mp-MRI-bx also identified 20 additional patients with intermediate/high-grade PCa that were either not detected or misclassified as low grade using TRUS-bx. Thus, pre-biopsy mp-MRI can increase the detection rate of significant PCa previously missed by TRUS-bx and stratify patients and lesions according to suspicion on mp-MRI.

In a targeted biopsy setting, the operator has to choose to either add the mp-MRI-bx to the systematic TRUS-bx scheme or rely on an mp-MRI-bx "targeted only" approach. Using mp-MRI-bx as an adjunct to TRUS-bx led to a GS upgrade in $37 \%$ of cases compared with TRUS-bx alone. Among these, $88 \%$ of patients (29/33) harbored intermediate/high-grade PCa and $38 \%(n=11)$ would not have been diagnosed with PCa if this had been based solely on TRUS-bx. Conversely, TRUS-bx identified PCa that were not detected using mp-MRI-bx, resulting in a $30 \%$ overall GS upgrade. However, the vast majority of these $(23 / 27$ [85\%]) were upgraded from no cancer on mpMRI-bx to GS 6 PCa on TRUS-bx. Therefore, mp-MRIbx increases the diagnoses of intermediate/high-grade cancers without increasing the diagnoses of low-grade disease.

Recent concerns have grown regarding the overdetection and subsequent overtreatment of men with insignificant low-grade PCa. The high diagnostic rate of lowgrade disease using TRUS-bx demonstrated in this study may add to these concerns and could favor a "targetedonly" strategy. Combining mp-MRI-bx with systematic TRUS-bx in our setting meant that 30 patients had to be 
biopsied to identify one additional man with GS $7(3+4)$ at the expense of 8 additional men with GS $6(3+3)$. Therefore, the combined strategy seems to be of limited clinical value in a repeated biopsy setting because the majority of patients with GS $\geq 7$ PCa were detected using mpMRI-bx, while TRUS-bx contributed a substantial number of patients with only low-grade disease. Overall, our results indicate that the diagnostic yield of intermediate/ high-grade vs. low-grade cancers can be improved using fewer biopsy cores if an mp-MRI "targeted-only" approach is applied. By only targeting patients with PI-RADS $\geq 3$ lesions, the number of men requiring biopsies and the diagnosis of low-grade PCa was reduced by 50 and $66 \%$, respectively. In addition, the detection of intermediate/ high-grade cancers increased by $52 \%$ compared with TRUS-bx. These results are consistent with a recent large study (1,003 patients) by Siddiqui et al. [21], which found that 200 patients had to be biopsied by TRUS-bx in addition to mp-MRI-bx to diagnose one additional patient with a high-risk tumor (GS $7[4+3]$ ), at the expense of 17 additional patients with low-risk tumors. However, that study included a mixed cohort of patients (biopsy-naive and men with prior negative TRUS-bx), excluded men with no abnormalities detected using mp-MRI, and defined low-volume GS 7(3+4) tumors as low-risk cancers. Moreover, the prospective study by Filson et al. [22] reported results that were contradicting to ours and found that the combined approach (mp-MRI-bx plus TRUS-bx) yielded the highest detection rate of significant cancer and result only in 1 additional low-risk PCa case detected per intermediate/high-risk PCa case. However, this study also included a mixed cohort of patients (biopsy-naive, prior negative, and prior positive biopsies) and the number of missed significant cancers following an mp-MRI "targeted-only" approach was significantly lower when considering only men with prior negative biopsies, as compared to our study. Nonetheless, the European Association of Urology guidelines [23] recommend a combined approach for all men with prior negative biopsies.

There are several limitations to this study. TRUS-bx and $\mathrm{mp}$-MRI results were interpreted by one highly experienced TRUS-operator and one dedicated mp-MRI physician. Less experienced operators might not achieve the same diagnostic yield. Our TRUS-bx technique for patients with prior negative TRUS-bx is modified to target the medial cores more anteriorly to sample a greater proportion of the anterior prostate, where tumors undetected by initial biopsies often reside. However, our repeated TRUS-bx scheme comprises the same prostatic sextant zones used in the initial biopsy setting and does not in- clude extended cores (>10-12 samples) or saturation-biopsies. Routinely increasing the number of cores might have improved the diagnostic rates at repeat TRUS-bx. However, it would not change the utility of mp-MRI-bx and insignificant PCa would still be detected.

The patients in this study were also selected as good candidates for targeted biopsies because many presented with rising PSA levels and clinical suspicion warranting a repeat biopsy. Therefore, these results may not be directly applicable to biopsy-naïve men, who constitute a different patient population. As a result, future studies in biopsy-naïve men such as the PRECISION trial (www. clinicaltrials.gov, NCT02380027) are evolving to clarify the implications of mp-MRI-bx and a "targeted-only" approach in the initial biopsy setting.

Additionally, because biopsy results were used comparatively in this study, the true rate of false negative readings cannot be assessed for patients with negative biopsies. A further analysis of patients who subsequently underwent RP following diagnosis might identify some cases, although this is a selected patient group. Using biopsy results as comparing reference allowed the comparison of 2 biopsy approaches in a repeat biopsy setting. Furthermore, previous studies have shown a good correlation between mp-MRI and the RP specimen [24, 25], even though some report that mp-MRI failed to detect significant tumors in 5-28\% of cases [26-28]. Thus, there is an unknown proportion of significant cancers that are difficult to detect using mp-MRI. In addition, prostate biopsies are still necessary to confirm the presence of $\mathrm{PCa}$ in a suspicious lesion, as mp-MRI findings are not cancerspecific. Furthermore, targeted mp-MRI-bx cannot always be accurate due to misregistration using image-fusion and PCa lesions may therefore be missed. Conversely, unnecessary targeted biopsies may be conducted due to false-positive mp-MRI readings. Moreover, the cost effectiveness of a diagnostic mp-MRI and the additional use of mp-MRI-bx have not been fully explored. Thus, cautions should be made about recommending "targeted-only" biopsies over a systematic approach. Nevertheless, our results indicate that cancers that are not detected by $\mathrm{mp}$-MRI followed by mp-MRI-bx in a repeat biopsy setting are unlikely to be significant higher-grade tumors that are detected using systematic repeated TRUS-bx cores.

Finally, the criterion for significant PCa was based solely on the GS from histopathological assessment of the biopsies and we chose not to include patient-specific static risk factors such as age, PSA, or number of visible MRI lesions, as they do not change between biopsy modalities. 
A number of definitions of insignificant and significant PCa including clinical findings, GS, and tumor volume (number of positive cores and maximum cancerous core length) have been proposed $[29,30]$. Including these parameters might have changed our results. However, there is still no clear consensus on the definition of significant $\mathrm{PCa}$ in targeted biopsies, although incorporating the maximum cancerous core length has been recommended for risk stratification [30]. Including only GS findings in the definition of significant PCa led to a more strict comparison between the 2 biopsy modalities.

In conclusion, mp-MRI/TRUS fusion biopsies have a high detection rate for significant $\mathrm{PCa}$ in patients with prior negative TRUS-bx and preferentially detect intermediate/high-grade compared with low-grade tumors. In patients with prior negative TRUS-bx and moderate to highly suspicious lesions on mp-MRI, an mp-MRI "targeted-only" biopsy strategy may be most beneficial. However, more comprehensive, multicenter studies will be required before recommending an mp-MRI "targeted-only” biopsy strategy for widespread adoption.

\section{Disclosure Statement}

The authors have no conflicts of interest to disclose.

\section{References}

1 Shariat SF, Roehrborn CG: Using biopsy to detect prostate cancer. Rev Urol 2008;10:262280.

2 Ploussard G, Nicolaiew N, Marchand C, Terry S, Vacherot F, Vordos D, et al: Prospective evaluation of an extended 21-core biopsy scheme as initial prostate cancer diagnostic strategy. Eur Urol 2014;65:154-161.

3 Maccagnano C, Gallina A, Roscigno M, Raber M, Capitanio U, Saccà A, et al: Prostate saturation biopsy following a first negative biopsy: state of the art. Urol Int 2012;89:126-135.

4 Ukimura O, Coleman JA, de la Taille A, Emberton M, Epstein JI, Freedland SJ, et al: Contemporary role of systematic prostate biopsies: indications, techniques, and implications for patient care. Eur Urol 2013;63:214-230.

5 Zaytoun OM, Moussa AS, Gao T, Fareed K, Jones JS: Office based transrectal saturation biopsy improves prostate cancer detection compared to extended biopsy in the repeat biopsy population. J Urol 2011;186:850-854.

6 Boccon-Gibod LM, Dumonceau O, Toublanc M, Ravery V, Boccon-Gibod LA: Micro-focal prostate cancer: a comparison of biopsy and radical prostatectomy specimen features. Eur Urol 2005;48:895-859.

7 Epstein JI, Feng Z, Trock BJ, Pierorazio PM: Upgrading and downgrading of prostate cancer from biopsy to radical prostatectomy: incidence and predictive factors using the modified Gleason grading system and factoring in tertiary grades. Eur Urol 2012;61:1019-1024.

8 Sciarra A, Barentsz J, Bjartell A, Eastham J, Hricak H, Panebianco V, et al: Advances in magnetic resonance imaging: how they are changing the management of prostate cancer. Eur Urol 2011;59:962-977.

9 Barentsz JO, Richenberg J, Clements R, Choyke P, Verma S, Villeirs G, et al: ESUR prostate MR guidelines 2012. Eur Radiol 2012;22:746-757.

10 Moore CM, Robertson NL, Arsanious N, Middleton T, Villers A, Klotz L, et al: Image- guided prostate biopsy using magnetic resonance imaging-derived targets: a systematic review. Eur Urol 2013;63:125-140.

11 Delongchamps NB, Peyromaure M, Schull A, Beuvon F, Bouazza N, Flam T, et al: Prebiopsy magnetic resonance imaging and prostate cancer detection: comparison of random and targeted biopsies. J Urol 2013;189:493-499.

12 Sonn GA, Chang E, Natarajan S, Margolis DJ, Macairan M, Lieu P, et al: Value of targeted prostate biopsy using magnetic resonance-ultrasound fusion in men with prior negative biopsy and elevated prostate-specific antigen. Eur Urol 2014;65:809-815.

13 Hambrock T, Hoeks C, Hulsbergen-van de Kaa C, Scheenen T, Fütterer J, Bouwense S, et al: Prospective assessment of prostate cancer aggressiveness using 3-T diffusion-weighted magnetic resonance imaging-guided biopsies versus a systematic 10 -core transrectal ultrasound prostate biopsy cohort. Eur Urol 2012; 61:177-184.

14 Siddiqui MM, Rais-Bahrami S, Truong $\mathrm{H}$, Stamatakis L, Vourganti S, Nix J, et al: Magnetic resonance imaging/ultrasound-fusion biopsy significantly upgrades prostate cancer versus systematic 12-core transrectal ultrasound biopsy. Eur Urol 2013;64:713-719.

15 Boesen L, Noergaard N, Chabanova E, Logager V, Balslev I, Mikines K, et al: Early experience with multiparametric magnetic resonance imaging-targeted biopsies under visual transrectal ultrasound guidance in patients suspicious for prostate cancer undergoing repeated biopsy. Scand J Urol 2015;49: 25-34.

16 Dickinson L, Ahmed HU, Allen C, Barentsz JO, Carey B, Futterer JJ, et al: Magnetic resonance imaging for the detection, localisation, and characterisation of prostate cancer: recommendations from a European consensus meeting. Eur Urol 2011;59:477-494.

17 Weinreb JC, Barentsz JO, Choyke PL, Cornud F, Haider MA, Macura KJ, et al: PI-RADS prostate imaging - reporting and data system: 2015, Version 2. Eur Urol 2016;69:16-40.

18 Epstein JI, Allsbrook WC Jr, Amin MB, Egevad LL: The 2005 international society of urological pathology (ISUP) consensus conference on gleason grading of prostatic carcinoma. Am J Surg Pathol 2005;29:1228-1242.

19 Epstein JI, Egevad L, Amin MB, Delahunt B, Srigley JR, Humphrey PA, et al: The 2014 international society of urological pathology (ISUP) consensus conference on gleason grading of prostatic Carcinoma: definition of grading patterns and proposal for a new grading system. Am J Surg Pathol 2016;40:244-252.

20 Vourganti S, Rastinehad A, Yerram NK, Nix J, Volkin D, Hoang A, et al: Multiparametric magnetic resonance imaging and ultrasound fusion biopsy detect prostate cancer in patients with prior negative transrectal ultrasound biopsies. J Urol 2012;188:2152-2157.

21 Siddiqui MM, Rais-Bahrami S, Turkbey B, George AK, Rothwax J, Shakir N, et al: Comparison of MR/ultrasound fusion - guided biopsy with ultrasound-guided biopsy for the diagnosis of prostate cancer. JAMA 2015;313: 390.

22 Filson CP, Natarajan S, Margolis DJ, Huang J, Lieu P, Dorey FJ, et al: Prostate cancer detection with magnetic resonance-ultrasound fusion biopsy: the role of systematic and targeted biopsies. Cancer 2016;122:884-892.

23 Mottet N, Bellmunt J, Bolla M, Briers E, Cumberbatch MG, De Santis M, et al: EAUESTRO-SIOG guidelines on prostate cancer. Part 1: screening, diagnosis, and local treatment with curative intent. Eur Urol 2017;71: 618-629.

24 Delongchamps NB, Lefèvre A, Bouazza N, Beuvon F, Legman P, Cornud F: Detection of significant prostate cancer with magnetic resonance targeted biopsies - should transrectal ultrasound-magnetic resonance imaging fusion guided biopsies alone be a standard of care? J Urol 2015;193:1198-1204. 
25 Turkbey B, Merino MJ, Gallardo EC, Shah $\mathrm{V}$, Aras O, Bernardo $\mathrm{M}$, et al: Comparison of endorectal coil and nonendorectal coil T2W and diffusion-weighted MRI at 3 Tesla for localizing prostate cancer: correlation with whole-mount histopathology. J Magn Reson Imaging 2014;39:14431448.

26 Tan N, Margolis DJ, Lu DY, King KG, Huang J, Reiter RE, et al: Characteristics of detected and missed prostate cancer foci on 3-T multiparametric MRI using an endorectal coil correlated with whole-mount thin-section histo- pathology. AJR Am J Roentgenol 2015; 205:W87-W92.

27 Thompson JE, van Leeuwen PJ, Moses D, Shnier R, Brenner P, Delprado W, et al: The diagnostic performance of multiparametric magnetic resonance imaging to detect significant prostate cancer. J Urol 2016;195:14281435.

28 Russo F, Regge D, Armando E, Giannini V, Vignati A, Mazzetti S, et al: Detection of prostate cancer index lesions with multiparametric magnetic resonance imaging (mp-MRI) using whole-mount histological sections as the reference standard. BJU Int 2016;118:8494.

29 Ploussard G, Epstein JI, Montironi R, Carroll PR, Wirth M, Grimm MO, et al: The contemporary concept of significant versus insignificant prostate cancer. Eur Urol 2011;60:291303.

30 Moore CM, Kasivisvanathan V, Eggener S, Emberton M, Fütterer JJ, Gill IS, Grubb RL, et al: Standards of reporting for MRI-targeted biopsy studies (START) of the prostate: recommendations from an International Working Group. Eur Urol 2013;64:544-552. 